\title{
Italique Italique
}

Poésie italienne de la Renaissance

XIV | 2011

Varia

\section{Lettura di «Ya siento el dulce espíritu de l'aura»di Fernando de Herrera}

Roland Béhar

\section{OpenEdition}

Journals

\section{Edizione digitale}

URL: http://journals.openedition.org/italique/331

DOI: 10.4000/italique.331

ISSN: 1663-4438

\section{Editore}

Librairie Droz

\section{Edizione cartacea}

Data di pubblicazione: 1 gennaio 2011

Paginazione: 101-115

ISBN: 978-2-600-01536-3

ISSN: 1423-3983

Notizia bibliografica digitale

Roland Béhar, « Lettura di «Ya siento el dulce espíritu de l'aura» di Fernando de Herrera », Italique [Online], XIV | 2011, online dal 24 juin 2014, consultato il 01 mai 2019. URL : http:// journals.openedition.org/italique/331 ; DOI : 10.4000/italique.331

(c) Tous droits réservés 


$$
\text { R OLAND BÉHAR }
$$

L E T T U R A

D I Y A S I E T T E L D ULCE ESPÍRITU

DE L'A UR A»

D I FER N N D O D E HERRERA 



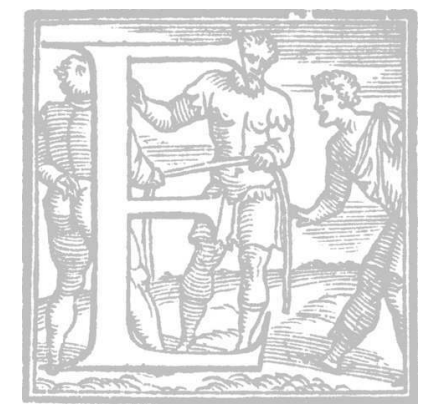

difficile parlare del petrarchismo di Fernando de Herrera (I534-1597) - «que al Petrarca desafía), secondo Lope de Vega - senza ricordare colui che, per il Cinquecento spagnolo, fu il nuovo Petrarca, e forse anche qualcosa di più: Garcilaso de la Vega (I499-I536). Spesso infatti si può leggere la volontà di Herrera di recuperare e superare l'ispirazione del poeta di Laura attraverso quella del toledano. Nel I580 Herrera dedica un monumentale commento a Don Antonio de Gurmán, marchese di Ayamonte, all'epoca governatore di Milano e capitano generale di tutta Italia: le Anotaciones a la poesía de Garcilaso, ${ }^{2}$ una specie di summa teorica che a volte - come gli è stato rimproverato - assomiglia più a delle polyantheae che non a un vero e proprio commento sulla poesia di Garcilaso. ${ }^{3}$

Due anni dopo Herrera dà alle stampe un canzoniere, Algunas obras de Fernando de Herrera, il cui titolo costituisce un ovvio riferimento a quello dell'edizione princeps di Garcilaso, pubblicata nel 1543: Las Obras de Boscán y algunas de Garcilaso. Non sapremo mai se l'incompintezza di quest'ultimo testo sia dovuta alla morte prematura di Garcilaso o all'oggettivo impianto dell'opera, che non è quello di un "canzoniere" petrarchista, ossia di una raccolta organica, un "macrotesto". A differenza del libro del I543, e con un'evidente volontà di emulazione, Herrera concepisce con sommo rigore la sua raccolta poetica, di chiarissimo stampo petrarchesco sin dal titolo: Algunas obras è un'eco delle "rime sparse" di Petrarca. Inoltre - e non è frequente in Spagna -, Herrera la pubblica, manifestando cosi la sua volontà d'imitatio. La sua poesia si muove lungo un cammino d'impostazione volutamente e quasi schematicamente petrarchista.

Frammentarie in modo soltanto apparente, le rime di Herrera sono dedicate alla sua donna, che cristallizza intorno a sé ogni bellezza mondana, sfoggiando diversi senhals il cui oggetto è costante: Luz, Estrella, Lumbre, Luzero, Eliodora o Aglaya. Tali senhals costringono l' "io" a identificarsi con Icaro o Fetonte, secondo un'immagine petrarchista ben conosciuta e già esplorata in tutti $i$ suoi aspetti poetici e meta-poetici dal Chariteo, dal Bembo e dal Tansillo. 
Nel I6rg viene pubblicata una nuova edizione: Versos de Fernando de Herrera emendados y dividos por él en tres libros (Siviglia, Gabriel Ramos Bejarano), dove il sonetto Ya siento el dulce espíritu de l'aura s'inserisce nel secondo libro. ${ }^{4}$ Inscritto nella trama di questo canzoniere, il nome di Luz riunisce in sé, come un microcosmo, l'intera storia del macrotesto: l'amore del poeta per l'amata Luz, nuova Laura. Rappresenta dunque il riassunto della narratio ( $" D e$ mi dichoso mal la rica istoria», come dice nel sonetto XXXVI, «Llevarme puede bien la suerte mia», v. I4), riassunto di una tradizione che non è soltanto petrarchista, ma anche elegiaca, per l'intenzione di tornare sulle orme dei maestri della poesia erotica antica, nella costante rememoratio o ruminatio del folle errore, del «vano error ${ }^{5}$ - cioè del furor degli elegiaci (cfr. Ovidio, Amores, I v. 35: "Blanditica comites tibi erunt errorque furorque»). Si tratta di una storia quasi senza racconto, ridotta alla sua quintessenza simbolica, alla magia del nome - Luz, che è il nome della Musa da cui il poeta è stato preso, affascinato, come lo fu Francesco (Secretum, III Is: «non minus nominis quam ipsius corporis splendore captus», eco di Properzio, I I "prima suis miserum me cepit ocellis»). ${ }^{6}$

La seconda parte dei Versos è inangurata dai versi:

El bello nombre quiere Amor que cante de mi Luz, por do, en propria o tierra agena, nunca otro español pie imprimió l'arena, siguiendo, Cintia i Delia, a vuestro amante.

L'imitatio, fondata sull'immagine dei passi del passato, è quella della grande tradizione elegiaca romana, non ancora sperimentata dagli spagnoli, e per la verità neanche da Garcilaso, nel senso in cui lo aveva fatto Luigi Alamanni. Come Petrarca lo è stato in Italia, Herrera ha la pretesa di essere il primo poeta elegiaco - dato che il suo compito è quello di cantare, evocare, celebrare la gloria di un solo nome: "Luz». In questo modo imita gli amanti delle muse eccelse, Cinzia e Delia, di cui scriveva nelle sue Anotaciones: «ambos an estado hasta aora tan iguales en grado que ninguno de los antiguos osó determinar quién era superiors. ${ }^{7}$

Ya siento el dulce espíritu de l'aura; que mansamente murmurando aspira; 
ya veo el puesto, a donde Amor me tira, $i$ ado su muerta llama el fuego instaura.

¿Cuál amador de Cintia, o Delia, o Laura temió más el desdén, l'ardiente ira; que yo la Luz, que tiernamente mira mi mal, i de la pena me restaura?

Como al qu'espantó el rayo con el trueno i lumbre; qu'aun le queda en la memoria el alto estruendo del terror passado;

tal yo, que estuve triste y siempre lleno de males, huyo en muestras de mi gloria, temiendo el bien que no esperé, engañado. ${ }^{8}$

Un primo asse di lettura di questo sonetto è dato dalla collazione delle rime, considerata come cornice semantica che sostiene un'architettura di frammenti d'imitazione petrarchesca non esente da ostentazione. Cio risulta particolarmente evidente nelle quartine: «l'aura» / «instaura» / «Laura» / «restaura»e, meno forte, «aspira» / «tira» / «ira» / «mira», seguite dalla coppia tanto decisamente petrarchesca della «memoria» e della «gloria», inscritta nel cuore finale delle terzine. Appaiono come essenziali anche $i$ due termini instaura $e$ restaura, simbolici di tutto un programma di recupero (restauratio) dell'estetica della fiamma petrarchista, creata dal poeta di Laura. Questa memoria petrarchesca del testo viene rinforzata dall'intreccio dei periodi testuali, ora caratterizzati da un'imitazione alla lettera di Petrarca, ora situati in una critica lontananza che riflette il desiderio di Herrera di scrivere nella prospettiva del "petrarchismo".

La prima quartina del sonetto si legge come l'evocatio quasi magica del nome della Musa di Petrarca. "Ya»: si tratta di un avverbio iniziale, di chiaro stampo petrarchesco e caro a Herrera, di cui troviamo altri esempi: in Petrarca (Rvf 33: Già fiammeggiava l'amorosa stella $e$ Rvf 217: Già desiai con sì giusta querela) e più ancora in Herrera, dove l'uso di questo avverbio meriterebbe un attento esame, soprattutto alla luce dell'uso ovidiano di iam e della riscrittura fattane da Garcilaso de la Vega nel sonetto XIII A Daphne ya los brazos le crecían. L'avverbio traduce dunque l'imminente arrivo della |laura| tanto desiderata, la vicinanza della musa petrarchista suprema. Il verso, e con lui il sonetto, si apre fra questi due elementi che fungono da cornice, concedendo loro la propria maestà. Allinterno della 
cornice (fra «Ya» e «l'aura») l'espressione «dulce espiritu» apre nella memoria del lettore cinquecentesco una vastissima serie di suggestioni intertestuali.

Il parallelo più evidente è quello con $\operatorname{Rvf} 320$ - in morte -:

Sento l'aura mia anticha, e i dolci colli veggio apparire, onde 'l bel lume nacque che tenne gli occhi mei mentr'al ciel piacque bramosi et lieti, or li ten tristi, et molli. ${ }^{9}$

A sua volta, esso ricorda l'altro incipit, adesso in vita ( $\operatorname{Rvf} 194)$ :

L'aura gentil, che rasserena i poggi destando i fiori per questo ombroso bosco, al soave suo spirto riconosco, per cui conven che 'n pena, e 'n fama poggi. ${ }^{\circ}$

Il riferimento, ovviamente, ̀̀ alla nota serie dell'aura, imperniata sul tema dell'identificazione di Laura con la brezza, in mezzo a una visione idillica della Provenza, locus amœnus della memoria innamorata - chiamata dal Contini «l'aura-situation». Tale serie presenta la storia, la narrazione dell'amore come appartenente a un passato chiuso. Riprendendo la situazione di Petrarca e ritornando a Valchiusa, Herrera finge a sua volta di ritrovare l'aura passata, ben conosciuta e temuta, di cui non sa se fidarsi o no.

La combinazione terminologica dei sonetti di Petrarca spiega dunque la scelta dei termini fatta da Herrera:

Ya [siento el dulce espíritu] de l'aura

Sento [...] l'aura mia anticha,

L'aura gentil [al soave suo spirto riconosco]

L'unica ripresa che non sia immediata è quella di dulce per soave, ben comprensibile se si pensa alla quasi sinonimia dei termini e al carattere petrarchesco tanto forte di questo epiteto. Del resto l'espressione riconosco indica ciò che è implicito ma ben presente nel sento di Petrarca e nel siento di Herrera: non è un atto di semplice percezione, di conoscenza, ma un atto di riconoscenza legato al ritorno al passato. Un'anagnorisis che definisce l' "io" un soggetto che percepisce Laura 
come Luz, cioè come oggetto di desiderio poetico, e vede se stesso come soggetto di un amore petrarchesco, ossia di una restauratio letteraria caratterizzata dal soffio dell'aura - la gloria.

Il secondo verso ("que mansamente murmurando aspira») si spiega con il ricordo di $\mathrm{Rvf}$ I96, generalmente letto come testo originale della serie petrarchesca dell'aura:

L'aura serena che fra verdi fronde mormorando a ferir nel volto viemme, fammi risovenir quand'amor diemme le prime piaghe, sì dolci, profonde. ${ }^{\text {I }}$

Ecco qui l'ultimo verbo derivato da Petrarca, che in questo caso assume la forma del gerundio: mormorando suggerisce murmurando, con l'aggiunta di un epiteto che non esiste in italiano ma che è stato nobilitato da Garcilaso nella sua imitazione degli Italiani: mansamente - la cui adlitteratio con murmurando permette un'ottima combinazione con il verbo di Petrarca (si vedano la Canción de Boscán Claros y frescos ríos / que mansamente vais, riscrittura del Chiare, fresche et dolci acque, e la celebre Canción III di Garcilaso Con manso ruido, echeggiata dall'Egloga II, vv. I-2 e I3: "En medio del invierno está templada / el agua dulce desta clara fuente [...] El dulce murmurar deste rü̈do»). Segne la seconda parte della quatina, con l'anafora di «ya»:

ya veo el puesto, a donde Amor me tira, i ado su muerta llama el fuego instaura.

Una perfetta simmetria con la prima parte della quartina è instaurata dalla reiterazione dello "ya» e, allo stesso tempo, dal ricordo del movimento originario di Petrarca:

Sento l'aura mia anticha, e i dolci colli

veggio apparire, onde 'l bel lume nacque.

L'alternanza tra il sentimento e la visione, già presente nell'ipotesto petrarchesco, è rinnovata e rinforzata dalla simmetria delle due parti della quartina. La descrizione che Herrera fa del luogo puesto è più astratta, e $i$ dolci colli cedono il passo al puesto, ma entrambi i poeti introducono una proposizione subordinata, raddoppiata nel caso di Herrera: 
onde 'l bel lume nacque

a donde Amor me tira,

i ado su muerta llama el fuego instaura.

Entrambi designano inoltre il luogo d'origine della passione e della poesia: origine che è nascita della luce per Petrarca, instauratio del fuoco della passione muerta llama per Herrera, ma che prepara anche la rima per la fine della seconda quartina: restaura.

Questa è senz'altro una nuova eco di Petrarca, piuttosto ovvia, perché ripresa dall'ultimo componimento della serie dell'aura, Rvf I97:

L'aura celeste che 'n quel verde lauro spira, ov'amor ferì nel fianco Apollo, et a me pose un dolce giogo al collo, tal che mia libertà tardi restauro. ${ }^{12}$

Abbiamo qui a che fare con l'evocazione della restauratio della libertà perduta dell'io, ma anche con l'emulatio di Herrera nella narratio della vicenda amorosa, più forte e sentita da lui che dagli altri poeti della tradizione - un altro locus del petrarchismo, già formulato dal Bembo nella sua famosa canzone A quai sembianza Amor Madonna agguaglia (Rime LXXIII), originariamente destinata agli Asolani, ma poi soppressa nella riscrittura dell'ancora più conosciuta Qual più diversa et nova di Petrarca Rvf I35:

Là dove 'l sol più tardo a noi s'adombra un vento si diparte, lo qual in ogni parte i boschi a suo spirar di fronde ingombra, che la fredda stagion dai rami sgombra. Così de lo mio core,

Ch'è selva di pensieri ombrosa e folta, Quand'ogni pace, ogni dolcezza è tolta, Però che sempre non consente Amore Ch'un uom per ben servir mieta dolore, Del suo dolce parlar lo spirto e l'aura Subitamente ogni mio mal restaura. ${ }^{\text {I }}$ 
Cinzia, Delia, Laura - Properzio, Tibullo, Petrarca: nomi simbolici di una poesia erotica sulle cui orme ritorna Herrera, con la volontà di restauratio, ma prendendo come tacita guida lo stesso Bembo.

Come definire l'originalità di Herrera? Con lo spostamento dell'oggetto della sua passione poetica dall'aura verso la luce. Non la Luz tranquilla, "que tiernamente mira», ma la Luce terribile del colpo di fulmine. Il cambio di oggetto sintetizza il cambio di poetica.

Questo spostamento è descritto in modo più preciso in un altro sonetto:

Aura mansa i templada d'Ocidente, que con el tierno soplo i blando frío halagas el ardor del pecho mío: ¿qu'espíritu te mueve vehemente?

$\mathrm{Ni}$ Euro espira ni Austro suena ardiente en el furor más grave del estío, i tú abrasas el verde prado i río, cual al suelo africano el sol caliente.

Mas, jai!, tú t'encendiste en mi Luz bella, $i$, enemiga del bien de mi ventura, abrasaste las ondas i las flores.

Cessa, Aura, no m'enciendas más, qu'en ella ardo siempre i me abraso en llama pura. ¡Ah!, no añadas más fuego a mis ardores. ${ }^{14}$

La complementarità delle immagini dell'aura e del raggio è già stata evidenziata dallo stesso Petrarca Rvf 90:

Erano i capei d'oro a l'aura sparsi che 'n mille dolci nodi gli avolgea, e 'l vago lume oltra misura ardea di quei begli occhi, ch'or ne son sì scarsi, e 'l viso di pietosi color farsi, non so se vero o falso mi parea. I' che l'esca amorosa al petto avea, qual meraviglia se di subito arsi?

Non era l'andar suo cosa mortale, ma d'angelica forma. Et le parole sonavan altro, che pur voce umana. 
Uno spirto celeste, un vivo sole fu quel ch'i' vidi. Et se non fosse or tale piaga per allentar d'arco non sana. ${ }^{\text {Is }}$

Sparsi nell'aura, ma uniti dall' amata nel suo stesso nome LAURA, i versi del poeta sono determinati dalla luce degli occhi, da quel «vago lume» che "oltre misura ardea» e che fece si che l' "io" "di subito ars[e]». "Spirto celeste» che soffia e dà ispirazione al poeta, illuminandolo con la Luce celeste della sua essenza. Spirito di cui si rammenta anche Herrera, ma rimanendone stupito (espantó):

Como al qu'espantó el rayo con el trueno i lumbre; qu'aun le queda en la memoria el alto estruendo del terror passado; tal yo, que estuve triste y siempre lleno de males, huyo en muestras de mi gloria, temiendo el bien que no esperé, engañado.

La tessitura delle terzine pare quasi riprendere una forma basata sulla similitudine, che funge spesso da cornice ai sonetti petrarcheschi. Come [...] così / Como [...] tal yo - forma che trova espressione nello stesso Garcilaso (sonetto XIX: Como la tierna madre que 'l doliente) e ancor più anticamente in Ausias March (Axí com cell qui 'n lo somnit 3 s' delita, Axí com cell qui desija vianda $o$ Axí com cell qui es veu prop de la mort) o in Jordi de Sant Jordí (Així com són sus l'espera los signes). ${ }^{16}$ Tradotta e imitata tante volte in castigliano, questa forma era stata integrata nella prassi poetica che coltivo con abbondanza il "sonetto comparativo". ${ }^{17}$ Tale similitudine indica il fatto che l'esperienza amorosa renda possibile lo sviluppo della parola poetica. Essa è paragonabile al fulmine, che lascia impressa nella memoria l'impronta della Luce / Luz; è un momento di pathos, un attimo più forte dalle tanto numerose metafore dell'amor pictor. ${ }^{18}$ Quest'imago agens potentissima permette a Herrera seguire un processo fondamentale per il petrarchismo, messo in luce da Antonio Daniele e, più recentemente, da Andrea Torre. ${ }^{19}$

L'immagine di cui Herrera fa uso gode, certo, di un'illustre tradizione elegiaca. Il furor amoroso deriva sempre, secondo il topos, dal fulgor degli occhi, come si vede nella canzone di Bembo. Con massima lucidità, Herrera ritorna, nel suo ricordo della storia avignonese di Francesco, 
alle vicende del primo stupor di fronte a Laura: stupor che lo fa chiamare Stupeus nel Bucolicum carmen, descritto nel Secretum $^{20}$ ed evocato tante volte nelle Rime, e stupor che troviamo nel ritratto della splendorosa Sofonisbe, nel quinto canto dell'Africa, forse archetipo della donna petrarchista.

Sarà lecito ricordare che Petrarca usa l'immagine dell'ictus fulminis anche in altri contesti, come nel passo della Canzone delle Visioni che simboleggia l'uccisione della donna amata, Rvf 323 (25-36):

In un boschetto novo, i rami santi fiorian d'un lauro giovenetto et schietto, ch'un delli arbor' parea di paradiso; et di sua ombra uscian sí dolci canti di vari augelli, et tant'altro diletto, che dal mondo m'avean tutto diviso; et mirandol io fiso, cangiossi ' 1 cielo intorno, et tinto in vista, folgorando 'l percosse, et da radice quella pianta felice súbito svelse: onde mia vita è trista, ché simile ombra mai non si racquista.

L'uccisione della donna amata non viene mai trattata nella storia di Herrera, dove il valore dello stupore è quello attribuito dall'adnominatio «error / furor / fulgor».

Dall'aura alla luce, grazie alla mediazione della meteorologia petrarchesca, Herrera opera uno spostamento: da soggetto del desiderio passionale e poetico diventa soggetto spaventato di fronte alla gloria evidente della sua Luce. Ma non può rammaricarsi, anche se nell'ultima terzina viene posto l'accento sulla tristezza, ossia sull'essenziale malinconia erotica (essenziale perché necessaria per la narratio): «tal yo, que estuve triste y siempre lleno / de males». La sofferenza manifesta la gloria, mentre la storia dell'errore passato eleva Fernando de Herrera più in alto di tutti $i$ suoi precedenti-Catullo, Tibullo, Petrarca.

Sembra lecito leggere questo errare finale dellio, engañado dalla paura di una Luce troppo forte, come trasposizione dell'estetica di una 
Spagna vincitrice e piena di gloria, le cui spoglie despojos adornano costantemente la poesia di Herrera. ${ }^{21}$ Malgrado numerose recusationes di Herrera stesso, il poeta viene considerato il cantore della vittoria di Lepanto. Il trionfo politico, manifestato dalla poesia epidittica, si riflette nell'atteggiamento verso la gloria divina, temuta, amata e adorata con riverenza, secondo la logica del potere del Nome.

Tutt'altra, e non paragonabile a quella di Herrera, sará la famosa riscrittura «dell'aura-situation» fatta dal contemporaneo Torquato Tasso, dove l'immediato e tanto desiderato arrivo dell'aura è reso ancora più evidente dal deittico dantesco ecco, di scarso uso nel Canzoniere di Petrarca. Tale deittico risveglia nella memoria del lettore il ricordo dell'aura in Dante, sempre congiunta col tremolar («inconcepibile in Petrarca», secondo il Contini). ${ }^{22}$ A rendere possibile questa nuova dispositio sono I) tanto la decisione di trascurare la presenza dell'io nel verbo, 2) quanto la scelta di aprire il componimento a una sistemazione paratattica degli elementi, innestati nella frase per riflettere non solo l'arrivo folgorante, ma anche la dolce epifania dell'amata - fonte di ogni luce, oriente ultimo:

Ecco mormorar l'onde

e tremolar le fronde

e l'aura mattutina e gli arboscelli, e sopra i verdi rami $i$ vaghi augelli cantar soavemente e rider l'oriente. ${ }^{23}$

Roland Béhar 
I. Cfr. Lope de Vega, Laurel de Apolo, ed., note, catalogo, indici di C. Giaffreda, introduzione di M. G. Profeti, Firenze, Alinea Editrice, 2002, p. I 36; Silva II, v. 385 .

2. [Obras de Garci Lasso con anotaciones de Fernando de Herrera al ilustrissimo i ecelentissimo Señor don Antonio de Guzman, Marques de Ayamonte, Governador del Estado de Milan, i Capitan General de Italia], Siviglia, Alonso de la Barrera, I 580.

3. Cfr. l'edizione con bibliografia sulla questione: Fernando de Herrera, Anotaciones a la poesía de Garcilaso, a cura di Inoria Pepe e José María Reyes, Madrid, Cátedra, $200 \mathrm{I}$.

4. La versione manoscritta del sonetto, quasi identica, ci assicura che l'autore è Herrera. Non entriamo qui nel lungo dibattito sul presunto autore delle varianti stilistiche: si tratta dello stesso Herrera, o di un suo discepolo - forse il pittore e storico della pittura Francisco Pacheco?

5. Cfr. ad esempio Algunas Obras, op. cit., 75.I-8: «Sigo por un desierto no tratado, / sin luz, sin guia, en confusion perdido, / el vano error, que solo m'à traido / a la miseria del mas triste estado. / Cuanto m'alàrgo mas, voi mas errado, / i a mayores peligros ofrecido. / dexar atras el mal m'es defendido; / qu'el passo del remedio està cerrado».

6. Francesco Petrarca, Prose, a cura di G. Martellotti, P. G. Ricci, E. Carrara e E. Bianchi, Milano, Sansoni, i955, p. I 58.

7. Fernando de Herrera, Anotaciones, p. 566.

8. Versos de Fernando de Herrera, p. 3 I6. Nelle edizioni moderne: Fernando DE Herrera, Poesía castellana original completa, edición de Cristóbal Cuevas, Madrid, Cátedra, I985, pp. 746-47. Le varianti del ms. I0.159 della BNE di Madrid sono indicate a p. 862. È stato José Manuel Blecua a portare alla luce questo ms., nell'edizione di Fernando de Herrera, Rimas inéditas, Madrid, CSIC, i 948, in questo caso p. 200 (con una maiuscola: Ya siento el dulçe espiritu de l'Aura) e alcune lezioni diverse (sopratutto di posizione) nelle terzine. Collazione ripresa nell'edizione: Fernando de Herrera, Obra poética, a cura di José Manuel Blecua, Madrid, Anejos del Boletín de la Real Academia Española, i 975, vol. II, p. 32 I.

9. Per il testo dei Rerum Vulgarium Fragmenta, cfr. Pétrarque, Chansonnier, a cura di G. Savoca, introduzione, traduzione e commento di G. Genot e F. Livi, Parigi, Les Belles Lettres, 2009, vol. I, p. 433.

io. Pétrarque, Chansonnier, p. 279.

i i. Pétrarque, Chansonnier, p. 28 I.

i 2. Pétrarque, Chansonnier, p. 283.

13. Pietro Bembo, Opere in volgare, a cura di Mario Marti, Firenze, Sansoni, ig6 i, p. 49I. 
i4. Fernando de Herrera, Poesía castellana original completa, a cura di Cristóbal Cuevas, Madrid, Cátedra, I985, p. 409.

i 5. Pétrarque, Chansonnier, p. i4 I.

i6. Una buona edizione, sebbene parziale, è quella di Ausiàs March, Páginas del Cancionero, introducción, edición y notas de Costanzo Di Girolamo, traducción José María Micó, Valencia, Editorial Pre-Textos, 2004.

17. Si veda Francesca dalle Pezze, Per una topologia sintattica del sonetto aureo spagnolo, Firenze, Alinea Editrice, 2002, pp. 53-67. Per i modelli di Herrera, possiamo menzionare Boscán (Como aquel que en soñar gusto recibe, Como, después del tempestoso día, Como el patrón que en golfo navegando) o Diego Hurtado de Mendoza (Como el hombre que buelga de soñar, Como el triste que á muerte es condenado, Como nave que corre en noche oscura).

I8. Per un caso della tradizione spagnola, con Garcilaso, cfr. Roland Béhar, «Lectura(s) de Garcilaso en el s. XVI: el caso del soneto v», in Compostella Aurea. Actas del VIII Congrero de la AJSO (Santiago de Compostele, 7-II de julio de 2008), coord. Antonio Azaustre Galiana e Santiago Fernández Mosquera, Santiago di Compostela, Universidade de Santiago de Compostela, 20 I I, pp. I 57-165.

19. Cfr. Antonio Daniele, La memoria innamorata. Indagini e letture petrarchesche, Roma-Padova, Editrice Antenore, 2005 e Andrea Torre, Petrarcheschi segni di memoria. Spie, postille, metafore, Pisa, Edizioni della Normale, 2007.

20. Francesco Petrarca vede la giovane Laura. L'importanza della visione nella descrizione del momento s'inscrive nella tradizione dell'innamoramento secondo il dolce stil novo, con la sua teoria pneumatologica degli «spirtì, di origine medicale. Il disastro si produce attraverso gli occhi, come in Properzio, I, i e, sopratutto, nell'Eneide, quando Virgilio descrive come Didone rimanga affascinata dall'immagine di Enea: «obstupuit primo aspectu sidonia Dido» (Eneide I 61 3). Di ciò troviamo ricordo nel Secretum, dove Sant'Agostino affianca il caso di Francesco a quello di Didone: «Habeo quod volebam. Obstupuisti, credo, perstrinxitque oculos fulgor insolitus. Dicunt enim stuporem amoris esse principium; binc est apud nature conscium poetam: obstupuit primo aspectu sidonia Dido. Post quod dictum sequitur: ardet amans Dido. Que quamvis, ut nosti optime, fabulosa narratio tota sit, ad nature tamen ordinem respexit ille, dum fingeret. Sed cum obstupuisses ad illius occursum, cur ad levam potissimum deflexisti?» (Francesco Petrarca, Prose, a cura di G. Martellotti, P. G. Ricci, E. Carrara e E. Bianchi, Milano-Napoli, Riccardo Ricciardi Editore, I955, p. I 5 2)

2 г. Cfr. Roland Béhar, «Los sagrados despojos de la veneranda antigüedad": estilo poético y debate literario en torno a Fernando de Herrera», in Josep Solervicens \& Antoni-Lluis Moll (a cura di), La poètica renaixentista a Europa. Una recreació del llegat clàssic, Barcellona, Punctum \& Mimesi, 201 I, pp. I 59-1 96.

22. Cfr. Peter Kuon, L'aura dantesca: metamorfosi intertestuali nei "Rerum Vulgarium Fragmenta" di Francesco Petrarca, Firenze, Franco Cesati, 2004. Per la citazione di Contini, cfr. Francesco Petrarca, Canzoniere, introduzione di Roberto Antonelli, Saggio di Gianfranco Contini, Note al testo di Daniele Ponchiroli, Torino, Einaudi, I992, p. XxxII. 
23. Torquato Tasso, Rime, a cura di Bruno Basile, Roma, Salerno, i 994, vol. I, pp. I 54-55 (libro II: Rime d'amore per Laura Peperara, $\mathrm{n}^{\circ}$ I 43). Vedasi, per questi versi, la lettura offerta da Salvatore Ritrovato nello stesso congresso della RSA di 2010. 